\title{
The independence polynomial of conjugate graph and noncommuting graph of groups of small order
}

\author{
Nabilah Najmuddin ${ }^{\mathrm{a},{ }^{*}}$, Nor Haniza Sarmin ${ }^{\mathrm{a}}$, Ahmad Erfanian ${ }^{\mathrm{b}}$ \\ ${ }^{a}$ Department of Mathematical Sciences, Faculty of Science, Universiti Teknologi Malaysia, 81310 UTM Johor Bahru, Johor, Malaysia \\ ${ }^{b}$ Department of Mathematics and Center of Excellence in Analysis on Algebraic Structures, Ferdowsi University of Mashhad, Azadi Square, \\ 9177948974 Mashhad, Razavi Khorasan, Iran \\ *Corresponding author: nabilah.najmuddin@yahoo.com
}

\section{Article history}

Received 13 June 2017

Accepted 5 November 2017

\section{Graphical abstract}

Some preliminaries on the conjugate graph and the noncommuting graph of groups of small order are stated.

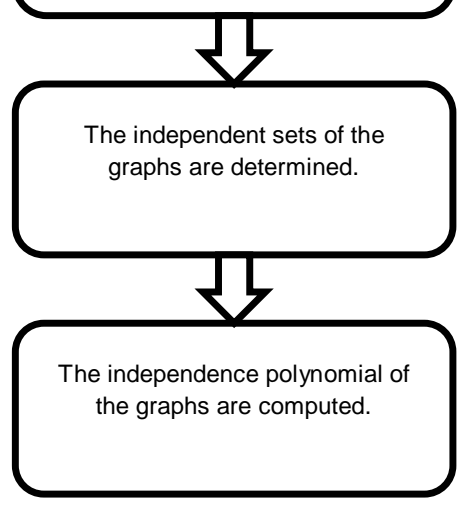

\begin{abstract}
An independent set of a graph is a set of pairwise non-adjacent vertices. The independence polynomial of a graph is defined as a polynomial in which the coefficient is the number of the independent set in the graph. Meanwhile, a graph of a group $G$ is called conjugate graph if the vertices are non-central elements of $G$ and two distinct vertices are adjacent if they are conjugate. The noncommuting graph is defined as a graph whose vertex set is non-central elements in which two vertices are adjacent if and only if they do not commute. In this research, the independence polynomial of the conjugate graph and noncommuting graph are found for three nonabelian groups of order at most eight.
\end{abstract}

Keywords: Independence polynomial, conjugate graph, noncommuting graph, dihedral group

\section{INTRODUCTION}

This section provides some backgrounds on graph theory and group theory. The groups stated in this paper are considered finite and non-abelian. There are many ways to associate a graph to a group. In this paper, only simple graphs are considered and will be referred only as graph.

Graph is the pair $\Gamma=(V, E)$ where $V$ is a nonempty set of vertices and $E$ is a set of unordered pair of elements of $V$, called the edges. A graph is simple if it has no loops and no multiple edges. If a graph $\Gamma=(V, E)$ and $u, v \in V$ then the vertices $u$ and $v$ are adjacent to each other in $\Gamma$ if and only if there is an edge between $u$ and $v$, i.e. $(u, v) \in E$. An edge $e=(x, y)$ is incident with each one of its end vertices, $x$ and $y$ [3].

In order to understand about independence polynomial, few definitions from graph theory are first stated here. An independent set is a set of vertices in a graph if no two vertices in the set are adjacent.
While the independence number of a graph $\Gamma$ is the maximum number of vertices in an independent set of vertices for the graph. This value is denoted as, $\alpha(\Gamma)$. [9].

A vertex $u$ is a neighbor of vertex $v$ in $G$ if $(u, v)$ is an edge of $\Gamma$. Open neighborhood (or just neighborhood), of . $v$. is defined to be the set of all vertices adjacent to $v$, denoted as $N(v)=\{u \in V \mid(u, v) \in E, u \neq v\}$. The set $N[v]=N(v) \cup\{v\}$ is the closed neighborhood of $v$ in $\Gamma$ [3]. If the neighborhood of every vertex is empty, means that there is no edge in the graph, then the graph is called empty graph, denoted by $E_{n}$. If $n=0$, then the graph is called null graph, denoted by $E_{0}:=\varnothing$.

Among the type of graph that is associated to group is the complete graph. A complete graph, denoted by $K_{n}$, is a graph on vertices where each pair of distinct vertices is connected by an edge. And other than that is the planar graph which is a graph that can be drawn in the plane without any edges crossing [9]. 
This paper is structured as follows: the first part is the introduction, the second part is the methodology used in this research while the third part includes the main results. We will compute the independence polynomial of the conjugate graph and the noncommuting graph of the groups $G_{1}, G_{2}$ and $G_{3}$. Given in the following:

(i) $\quad G_{1}=\left\langle a, b: a^{3}=b^{2}=1, b a b=a^{-1}\right\rangle$

$$
G_{2}=\left\langle a, b: a^{4}=b^{2}=1, b a b=a^{-1}\right\rangle
$$

$$
G_{3}=\left\langle a, b: a^{4}=1, b^{2}=a^{2}, a b a=b\right\rangle .
$$

Note that $G_{1}$ is the dihedral group of order $6, G_{2}$ is the dihedral group of order 8 , and $G_{3}$ is the quaternion group of order 8 .

\section{PRELIMINARIES}

In this section, some basic concepts in graph theory that are related to group theory are included. We can compute the independence polynomial of the graphs using some methods that will also be provided in this section. The following are some basic concepts on independence polynomial, that we will be using throughout this paper.

Definition 2.1 [10] Independence Polynomial

The independence polynomial of a graph $\Gamma$ is the polynomial whose coefficient on $x^{k}$ is given by the number of independent sets of order $k$ in $\Gamma$. This is denoted by $I(\Gamma ; \mathrm{x})$. So

$$
I(\Gamma ; \mathrm{x})=\sum_{k=0}^{\alpha(\Gamma)} c_{k} x^{k}
$$

where $c_{k}$ is the number of independent sets of order $k$ in $\Gamma$.

Definition 2.2 [10] Independence Polynomial of Null Graph The independence polynomial of null graph is $I(\varnothing ; x)=1$.

\section{Proposition 2.1 [10]}

The independence polynomial of the single vertex graph, known as singleton, is given by $1+x$.

The following theorems are the methods used by Hoede and Li [6] and Ferrin [4] in calculating the independence polynomial for various families of graphs.

\section{Theorem 2.1 [10]}

Let $\Gamma_{1}$ and $\Gamma_{2}$ be two disjoint graphs. Then $I\left(\Gamma_{1} \cup \Gamma_{2} ; x\right)=I\left(\Gamma_{1} ; x\right) \cdot I\left(\Gamma_{2} ; x\right)$.

Theorem 2.2 [10]

Let $\Gamma$ be a simple graph and $v \in V$. Then $I(\Gamma ; x)=I(\Gamma-v: x)+x I(\Gamma-N[v] ; x)$.

Theorem 2.3 [10]

Let $\Gamma$ be a simple graph and $e=(u, v) \in E$.

Then $I(\Gamma ; x)=I(\Gamma \backslash e: x)-x^{2} I(\Gamma-(N[u] \cup N[v]) ; x)$.

In order to compute the independence polynomial of some common graphs, Ferrin [8] had used Theorem 2.1, 2.2 and 2.3 together with the concept stated in Proposition 2.1 and also with the propositions stated below.

\section{Proposition 2.2 [8]}

The independence polynomial of an empty graph $\Gamma$ of order $n$ is given by $I(\Gamma ; x)=(1+x)^{n}$.
Proposition 2.3 [8]

The independence polynomial of complete graph, $K_{n}$ is $I\left(K_{n} ; x\right)=n x+1$.

Next, we state some basic concepts from group theory and graph theory that mostly are related to conjugate graph and noncommuting graph that are used in this paper.

Definition 2.3 [6] Conjugacy Class

Let $G$ be a group and $a, x \in G$. For a fixed element $a \in G$, the conjugacy class of $a$ in $G$ is $c l(a)=\left\{g \in G\right.$ :there exists $\left.x \in G, g=x a x^{-1}\right\}$. Also, $x^{-1}$ is called the conjugate of $a$ by $x$ in the group $G$.

\section{Proposition 2.4 [6]}

Let $G$ be a group and $a, b \in G$. If $a$ and $b$ belong in one conjugacy class, that is $\operatorname{cl}(a)=\operatorname{cl}(b)$, then elements $a$ and $b$ are conjugate to each other.

\section{Proposition 2.5 [6]}

Let $a$ be an element in $G$. If the conjugacy class of $a$ contains only one element, then $a$ lies in the center $Z(G)=\{y \in G: x y=y x, x \in G\}$.

Definition 2.4 [6] Conjugate Graph

A conjugate graph $\Gamma_{G}^{c}$ of a group $G$, is defined as the graph whose vertex set, $V\left(\Gamma_{G}^{c}\right)$ is non-central elements of $G$, that is $\left|V\left(\Gamma_{G}^{c}\right)\right|=|G|-|Z(G)|$ in which two distinct vertices are adjacent if they are conjugate.

\section{Theorem 2.4 [19]}

Let $G_{1}$ be the dihedral group of order 6, $G_{1}=\left\langle a, b: a^{3}=b^{2}=1, b a b=a^{-1}\right\rangle$. The conjugacy classes of elements of $\quad G_{1} \quad$ are $\quad c l(e)=\{e\}, \quad c l(a)=\left\{a, a^{2}\right\}=\operatorname{cl}\left(a^{2}\right) \quad$ and $c l(b)=\left\{b, a b, a^{2} b\right\}=c l(a b)=\operatorname{cl}\left(a^{2} b\right)$. Then, the conjugate graph of $G_{1}$ is the union of complete graph $K_{2}$ and $K_{3}$, denoted as $\Gamma_{G_{1}}^{c}=K_{2} \cup K_{3}$ as shown in Figure 1.
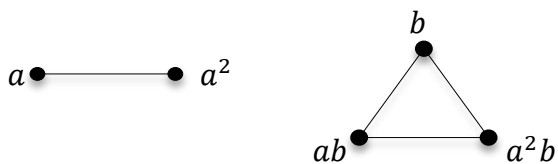

Figure 1 The Conjugate Graph of $\boldsymbol{G}_{1}, \Gamma_{\boldsymbol{G}_{1}}^{c}$

Theorem 2.5 [19]

Let $G_{2}$ be the dihedral group of order 8, $G_{2}=\left\langle a, b: a^{4}=b^{2}=1, b a b=a^{-1}\right\rangle$. The conjugacy classes of elements of $G_{2} \quad$ are $\quad c l(e)=\{e\}, \quad c l\left(a^{2}\right)=\left\{a^{2}\right\}, \quad c l(a)=\left\{a, a^{3}\right\}=c l\left(a^{3}\right)$, $c l(b)=\left\{b, a^{2} b\right\}=\operatorname{cl}\left(a^{2} b\right)$ and $\operatorname{cl}(a b)=\left\{a b, a^{3} b\right\}=\operatorname{cl}\left(a^{3} b\right)$. Then the conjugate graph of $G_{2}$ is the union of three complete graphs $K_{2}$, denoted as $\Gamma_{G_{2}}^{c}=K_{2} \cup K_{2} \cup K_{2}$ as shown in Figure 2 .

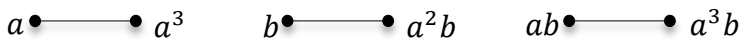

Figure 2 The Conjugate Graph of $\boldsymbol{G}_{2}, \boldsymbol{\Gamma}_{\boldsymbol{G}_{2}}^{\boldsymbol{c}}$ 
Theorem 2.6 [19]

Let $G_{3}$ be the quaternion group of order 8, $G_{3}=\left\langle a, b: a^{4}=1, b^{2}=a^{2}, a b a=b\right\rangle$. The conjugacy classes of $G_{3}$ is the same as the conjugacy classes in Theorem 1.4. Then, the conjugate graph of $G_{3}, \Gamma_{G_{3}}^{c}$ is isomorphic with the conjugate graph in Figure 2.

\section{Definition 2.5 [1] Noncommuting Graph}

A noncommuting graph $\Gamma_{G}^{n c}$ of a group $G$ is defined as the graph whose vertex set, $V\left(\Gamma_{G}^{n c}\right)$ is non-central elements of $G$, in which two distinct vertices are adjacent if and only if they do not commute.

\section{Theorem 2.7 [1]}

Let $G_{1}$ be the dihedral group of order 6 , $G_{1}=\left\langle a, b: a^{3}=b^{2}=1, b a b=a^{-1}\right\rangle$. Then the noncommuting graph of $G_{1}$ is a planar graph of 5 vertices in Figure 3.

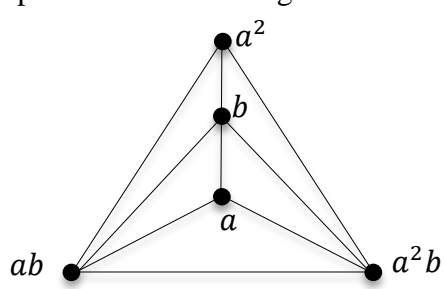

Figure 3 The Noncommuting Graph of $\boldsymbol{G}_{1}, \boldsymbol{\Gamma}_{\boldsymbol{G}_{1}}^{n \boldsymbol{c}}$

\section{Theorem 2.8 [1]}

Let $G_{2}$ be the dihedral group of order 8 , $G_{2}=\left\langle a, b: a^{4}=b^{2}=1, b a b=a^{-1}\right\rangle$. Then the noncommuting graph of $G_{2}$ is a planar graph of 6 vertices in Figure 4.

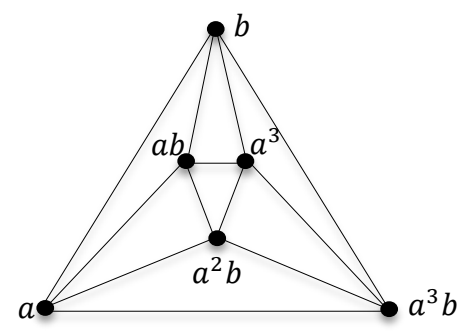

Figure 4 The Noncommuting Graph of $\boldsymbol{G}_{2}, \Gamma_{\boldsymbol{G}_{2}}^{n \boldsymbol{c}}$

\section{Theorem 2.9 [1]}

Let $G_{3}$ be the quaternion group of order 8 , $G_{3}=\left\langle a, b: a^{4}=1, b^{2}=a^{2}, a b a=b\right\rangle$. The elements of $G_{3}$ that do not commute are the same as the elements that do not commute in $G_{2}$ in Theorem 1.8. Hence, the noncommuting graph of $G_{3}, \Gamma_{G_{3}}^{n c}$ is also a planar graph with 6 vertices like in Figure 4.

The aim of this paper is to obtain the independence polynomial of the conjugate graph for the nonabelian groups of order at most 8 which are $\Gamma_{G_{1}}^{c}, \Gamma_{G_{2}}^{c}$ and $\Gamma_{G_{3}}^{c}$ and also the noncommuting graphs $\Gamma_{G_{1}}^{n c}$ , $\Gamma_{G_{2}}^{n c}$ and $\Gamma_{G_{3}}^{n c}$.

\section{MAIN RESULTS}

This section consists of two parts. The first part presents the finding on the independence polynomial of the conjugate graph of groups $G_{1}$, $G_{2}$ and $G_{3}$. While the second part presents the independence polynomial of the noncommuting graph of the same groups as the first part.

\section{The Independence Polynomial of Conjugate Graph of Nonabelian Groups of Order At Most Eight}

This is the first part of the main result in which the independence polynomials of conjugate graph of groups $G_{1}, G_{2}$ and $G_{3}$ are obtained.

Theorem 3.1 Let $G_{1}$ be the dihedral group of order 6, $G_{1}=\left\langle a, b: a^{3}=b^{2}=1, b a b=a^{-1}\right\rangle$. Then the independence polynomial of the conjugate graph of $G_{1}$ is $I\left(\Gamma_{G_{1}}^{c} ; x\right)=1+5 x+6 x^{2}$.

Proof: The independence number of graph $\Gamma_{G_{1}}^{c}$ is $\alpha\left(\Gamma_{G_{2}}^{c}\right)=2$. There are six independent vertex sets with order 2 which are $\{a, b\},\{a, a b\}$, $\left\{a, a^{2} b\right\},\left\{a^{2}, b\right\},\left\{a^{2}, a b\right\}$ and $\left\{a^{2}, a^{2} b\right\}$, and five independent vertex sets with order 1 in which each set contain each vertex of $G_{1}$ denoted as $\{a\},\left\{a^{2}\right\},\{b\},\{a b\}$ and $\left\{a^{2} b\right\}$. Then, by Definition 2.1 and 2.2 , we obtain

$$
I\left(\Gamma_{G_{1}}^{c} ; \mathrm{x}\right)=\sum_{k=0}^{2} c_{k} x^{k}=c_{0} x^{0}+c_{1} x^{1}+c_{2} x^{2}=1+5 x+6 x^{2} .
$$

Theorem 3.2 Let $G_{2}$ be the dihedral group of order 8, $G_{2}=\left\langle a, b: a^{4}=b^{2}=1, b a b=a^{-1}\right\rangle$. Then the independence polynomial of the conjugate graph of $G_{2}$ is $I\left(\Gamma_{G_{2}}^{c} ; x\right)=1+6 x+12 x^{2}+8 x^{3}$.

Proof The graph $\Gamma_{G_{2}}^{c}$ has independence number $\alpha\left(\Gamma_{G_{2}}^{c}\right)=3$. From the graph, there are eight independent vertex sets with order 3 which are $\{a, b, a b\},\left\{a, b, a^{3} b\right\},\left\{a, a^{2} b, a b\right\},\left\{a, a^{2} b, a^{3} b\right\},\left\{a^{3}, b, a b\right\}$, $\left\{a^{3}, b, a^{3} b\right\},\left\{a^{3}, a^{2} b, a b\right\}$ and $\left\{a^{3}, a^{2} b, a^{3} b\right\}$. The independent vertex sets with order two are $\{a, b\},\{a, a b\},\left\{a, a^{2} b\right\},\left\{a, a^{3} b\right\},\left\{a^{3}, b\right\}$, $\left\{a^{3}, a b\right\},\left\{a^{3}, a^{2} b\right\},\left\{a^{3}, a^{3} b\right\},\{b, a b\},\left\{b, a^{3} b\right\},\left\{a^{2} b, a b\right\}$ and $\left\{a^{2} b, a^{3} b\right\}$ and independent vertex sets with order one are each set containing each vertex of $G_{2}$ denoted as $\{a\},\left\{a^{3}\right\},\{b\},\{a b\}$, $\left\{a^{2} b\right\}$ and $\left\{a^{3} b\right\}$. Hence, by Definition 2.1 and 2.2,

$$
I\left(\Gamma_{G_{2}}^{c} ; \mathrm{x}\right)=\sum_{k=0}^{3} c_{k} x^{k}=c_{0} x^{0}+c_{1} x^{1}+c_{2} x^{2}+c_{3} x^{3}=1+6 x+12 x^{2}+8 x^{3} \cdot \square
$$

Theorem 3.3 Let $G_{3}$ be the quaternion group of order 8, $G_{3}=\left\langle a, b: a^{4}=1, b^{2}=a^{2}, a b a=b\right\rangle . \quad I\left(\Gamma_{G_{3}}^{c} ; x\right)=1+6 x+12 x^{2}+8 x^{3}$ is the independence polynomial of the conjugate graph of $G_{3}$.

Proof Since the conjugate graph of $G_{3}, \Gamma_{G_{3}}^{c}$ is isomorphic with the conjugate graph of $G_{2}, \Gamma_{G_{2}}^{c}$, hence the proof follows from the proof of Theorem 3.2. $\square$ 


\section{The Independence Polynomial of Noncommuting Graph of Nonabelian Groups of Order At Most Eight}

The second part of our main result is the independence polynomial of non-commuting graph of the groups $G_{1}, G_{2}$ and $G_{3}$.

Theorem 4.1 Let $G_{1}=\left\langle a, b: a^{3}=b^{2}=1, b a b=a^{-1}\right\rangle$. Then the independence polynomial of the noncommuting graph of $G_{1}$ is $I\left(\Gamma_{G_{1}}^{n c} ; x\right)=1+5 x+x^{2}$.

Proof Based on Definition 1.5, the independence number of $\Gamma_{G_{1}}^{n c}$ is 2 . There is only one independent vertex sets with order 2 which is $\left\{a, a^{2}\right\}$. And there are five independent vertex sets with order 1 which are $\{a\},\left\{a^{2}\right\},\{b\},\{a b\}$ and $\left\{a^{2} b\right\}$. Again, by Definition 2.1 and 2.2, we obtain

$$
I\left(\Gamma_{G_{1}}^{n c} ; x\right)=\sum_{k=0}^{2} c_{k} x^{k}=c_{0} x^{0}+c_{1} x^{1}+c_{2} x^{2}=1+5 x+x^{2} \cdot \square
$$

Theorem 4.2 Let $G_{2}=\left\langle a, b: a^{4}=b^{2}=1, b a b=a^{-1}\right\rangle$. Then the independence polynomial of the noncommuting graph of $G_{2}$ is $I\left(\Gamma_{G_{2}}^{n c} ; x\right)=1+6 x+3 x^{2}$.

Proof The graph $\Gamma_{G_{2}}^{n c}$ has independence number $\alpha\left(\Gamma_{G_{2}}^{n c}\right)=2$. From the graph, there are three independent vertex sets with order two which are $\left\{a, a^{3}\right\},\left\{b, a^{2} b\right\}$. and $\left\{a b, a^{3} b\right\}$, and the independent vertex sets with order one are each set containing each vertex of $G_{2}$ denoted as $\{a\},\left\{a^{3}\right\},\{b\},\{a b\},\left\{a^{2} b\right\}$ and $\left\{a^{3} b\right\}$. Hence, by Definition 2.1 and 2.2,

$$
I\left(\Gamma_{G_{2}}^{n c} ; \mathrm{x}\right)=\sum_{k=0}^{2} c_{k} x^{k}=c_{0} x^{0}+c_{1} x^{1}+c_{2} x^{2}=1+6 x+3 x^{2} .
$$

Theorem 4.3 Let $G_{3}=\left\langle a, b: a^{4}=1, b^{2}=a^{2}, a b a=b\right\rangle$. Then the independence polynomial of the noncommuting graph of $G_{3}$, $I\left(\Gamma_{G_{3}}^{n c} ; x\right)=1+6 x+3 x^{2}$.

Proof Since the noncommuting graph of $G_{3}, \Gamma_{G_{3}}^{n c}$ is isomorphic with the noncommuting graph of $G_{2}, \Gamma_{G_{2}}^{n c}$, then the proof follows from the proof of Theorem 4.2.

\section{CONCLUSION}

In this paper, the independence polynomial of the conjugate graph and the non-commuting graph of three groups of small order are computed. For the group $G_{1}$, the independence polynomial of its conjugate graph is $I\left(\Gamma_{G_{1}}^{c} ; x\right)=1+5 x+6 x^{2}$ and the independence polynomial of its noncommuting graph is $I\left(\Gamma_{G_{1}}^{n c} ; x\right)=1+5 x+x^{2}$. For group $G_{2}, I\left(\Gamma_{G_{2}}^{c} ; x\right)=1+6 x+12 x^{2}+8 x^{3} \quad$ is the independence polynomial of the conjugate graph and $I\left(\Gamma_{G_{2}}^{n c} ; x\right)=1+6 x+3 x^{2}$ is the independence polynomial of the noncommuting graph. And for the third group $G_{3}$, the independence polynomial of its conjugate graph is
$I\left(\Gamma_{G_{3}}^{c} ; x\right)=1+6 x+12 x^{2}+8 x^{3}$ and $I\left(\Gamma_{G_{3}}^{n c} ; x\right)=1+6 x+3 x^{2}$ is the independence polynomial of its noncommuting graph. Since the conjugate graph of $G_{2}$ and the noncommuting graph of $G_{2}$ are isomorphic to the conjugate graph of $G_{3}$ and the noncommuting graph of $G_{3}$, respectively, therefore the independence polynomial of the conjugate graph and the non-commuting graph are the same for both groups.

\section{ACKNOWLEDGEMENT}

The first author would like to thank Universiti Sains Malaysia and the Ministry of Higher Education for the full scholarship given.

\section{REFERENCES}

[1] Abdollahi, A., S. Akbari, and H. R. Maimani. 2006. Non-Commuting Graph of a Group. Journal of Algebra. 298(2006): 468-492.

[2] Asghar Talebih, A. 2008. On the Non-commuting Graphs of Group $D_{2 n}$ International Journal of Algebra. 2(20): 957-961.

[3] Balakrishnan, R. and K. Ranganathan. 2012. A Textbook of Graph Theory. $2^{\text {nd }}$ ed. New York: Springer.

[4] Bertram, E. A. 1983. Some Applications of Graph Theory to Finite Groups. Discrete Mathematics. 44: 31-43.

[5] Darafsheh, M. R. 2009. Groups with the Same Non-commuting Graph. Discrete Applied Mathematics. 157: 833-837.

[6] Erfanian, A. and B. Tolue. 2012. Conjugate Graphs of Finite Groups. Discrete Mathematics, Algorithms and Applications. 4(2): 35-43.

[7] Erfanian, A., F. Mansoori and B. Tolue. 2015. Generalized Conjugate Graph. Georgian Mathematical Journal. 22(1): 1-8.

[8] Ferrin, G. 2014. Independence Polynomials. Master Dissertation. University of South Carolina.

[9] Fraleigh, J. B. 2003. A First Course in Abstract Algebra. $7^{\text {th }}$ ed. U.S.A.: Pearson Education, Inc.

[10] Hoede, C. and X. Li. 1994. Clique Polynomials and Independent Set Polynomials of Graphs. Discrete Mathematics. 125: 219-228.

[11] Levit, V. E. and E. Mandrescu. 2005. The Independence Polynomial of a Graph - A Survey. Proceedings of the $1^{\text {st }}$ International Conference on Algebraic Informatics. 233254.

[12] Moghaddamfar, A. R., W. J. Shi, W. Zhou and A. R. Zokayi. 2005. On the Noncommuting Graph Associated with a Finite Group. Sherian Mathematical Journal. 46(2): 325-332.

[13] Moradipour K., N. H. Sarmin and A. Erfanian. 2013. On Noncommuting Graphs of Some Finite Groups. International Jrnal of Applied Mathematics and Statistics. 45(15): 473-476.

[14] Omer, S. M. S., N. H. Sarmin and A. Erfanian. 2013. The Probability that an Element of a Group Fixes a Set and Its Graph Related to Conjugacy Classes. Journal of Basic Applied Scientific Research. 3(10): 369-380.

[15] Omer, S. M. S., N. H. Sarmin and A. Erfanian. 2013. The Probability that an Element of a Symmetric Group Fixes a Set and Its Application in Graph Theory. World Applied Sciences Journal. 27(12): 1637-1642.

[16] Rose, H. E. 2009. A Course on Finite Groups. London: Springer-Verlag.

[17] Rosen, K. H. 2013. Discrete Mathematics and Its Applications. $7^{\text {th }}$ ed. New York: McGraw-Hill.

[18] Rotman, J. J. 2003. Advanced Modern Algebra. USA: Prentice Hall.

[19] Sarmin, N. H., I. Gambo and S. M. S. Omer. 2015. The Conjugacy Classes of Metabelian Groups of Order at Most 24. Jurnal Teknologi. 77(1) : 139-143.

[20] Sarmin, N. H., M. Jahandideh and M. R. Darafsheh. 2016. Recent Advances in Commutativity Degrees and Graphs of Groups. Chapter 1: 1-8. Penerbit UTM Press.

[21] Sarmin, N. H., N. H. Bilhikmah, S. M. S. Omer and A. H. M. Noor. 2016. The Conjugacy Classes, Conjugate graph and Conjugacy Class Graph of Some Finitie Metacyclic 2-groups. Menemui Matematik. 38(1): 1-12. 